Culture et histoire dans l'espace roman

$12 \mid 2014$

Empreintes/emprunts : entre forces de conformisation et forces d'innovation

\title{
Réemprunts et prégnance du décor dans les films historiques espagnols
}

\section{Nicolas Blayo}

\section{(2) OpenEdition}

\section{Journals}

Édition électronique

URL : https://journals.openedition.org/cher/7602

DOI : $10.4000 /$ cher.7602

ISSN : 2803-5992

Éditeur

Presses universitaires de Strasbourg

Édition imprimée

Date de publication : 7 juillet 2014

Pagination : $35-43$

ISBN : 978-2-86820-569-8

ISSN : 1968-035X

Référence électronique

Nicolas Blayo, "Réemprunts et prégnance du décor dans les films historiques espagnols »,

reCHERches [En ligne], 12| 2014, mis en ligne le 13 décembre 2021, consulté le 15 décembre 2021.

URL : http://journals.openedition.org/cher/7602 ; DOI : https://doi.org/10.4000/cher.7602

\section{(c) (i) (2)(2)}

Ce(tte) œuvre est mise à disposition selon les termes de la Licence Creative Commons Attribution -

Pas d'Utilisation Commerciale - Partage dans les Mêmes Conditions 4.0 International. 


\title{
Réemprunts et prégnance du décor dans les films historiques espagnols
}

\author{
NicoLAS BLAYO \\ Université de Lorraine - Metz
}

Depuis la fin de 1975 jusqu'en 2011, on compte en Espagne une centaine de films historiques consacrés aux années comprises entre 1936 et 1975. Ces films couvrent tous les genres, drame, comédie, polar, musical, roadmovie, fantastique, etc., même si l'on observe une très nette prédilection pour la comédie et le drame. Si le scénario a une place prépondérante, il est bien évident que la conception même de film historique passe aussi par les décors destinés à offrir au spectateur une représentation du passé, mais les décors qui situent l'action dans le temps sont «surtout là pour lui conférer une authenticité»(Puaux 2008: 17). Et c'est bien là que les difficultés commencent car la représentation du passé va poser un problème à tout réalisateur - et c'est l'immense majorité - qui voudra obtenir le plus grand réalisme possible. La question du réalisme des films historiques renvoie à une représentation qui serait fidèle au passé, vraisemblable et acceptable par le spectateur qui accepterait le pacte que lui proposerait tacitement le réalisateur de sorte qu'il n'y aurait aucune espèce de doute, de débat ou de remise en cause du passé ainsi reconstitué par les décorateurs.

De quelle nature est le film historique? Ce n'est pas un récit historique à visée savante - on aura le documentaire historique pour cela -, ce n'est pas non plus une fiction où tout serait inventé - et alors que resterait-t-il d'historique? - c'est bien, à des degrés divers, un récit fictionnel dans lequel l'histoire entre comme ingrédient de la fiction, c'est pourquoi on a pu, avec Paul Ricœur, parler d'une double articulation d'historicisation de la fiction et de fictionnalisation de l'histoire (Ricœur 1985: 329). Cette imbrication

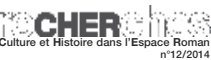


d'éléments historiques et fictionnels dans un récit filmique pose d'autres défis et d'autres enjeux que le récit historique littéraire, qui a inspiré Georg Lukács dans la première réflexion théorique sur le roman historique. Le film est un art audio-visuel qui doit transcrire par l'image et le son la dimension historique qu'il veut donner au récit fictionnel. Nous avons traité ailleurs d'un aspect de la partie sonore et en particulier de l'importance capitale de la récupération de la copla, la chanson populaire, dans les films historiques (Blayo 2010: 133) et nous nous intéresserons ici à l'aspect visuel du film d'époque.

L'élaboration de tous les aspects visuels du film incombe à celui que l'on a coutume d'appeler le directeur artistique, et qui va avoir un rôle majeur dans la construction du film, ainsi que propose de le définir le cinéaste Jaime Camino:

En su sentido más amplio es quien concibe, planea y elige el espacio en que va a desarrollarse la acción del film y los elementos contenidos en ese espacio. [...] Embebido del guión y del estilo que el director quiere imprimir a su película, el director artístico dibujará los decorados que deban construirse (o controlará la labor del decorador), elegirá con el director los interiores y exteriores naturales, trabajará con el figurinista - caso de no ser el propio D. A. quien diseñe el vestuario - y controlará en general todos los aspectos visuales del film. (Camino 1999: 34).

On voit dans cette définition l'amplitude d'une spécialité qui, en dépit de l'adjectif «artístico» est couramment considérée comme relevant de l'équipe technique du film par opposition à l'équipe artistique composée essentiellement des interprètes. Le directeur artistique, titre que certains décorateurs prestigieux comme Gil Parrondo estiment exagérément pompeux par rapport à celui de décorateur qui s'employait autrefois, a bel et bien un rôle qui dépasse largement le décor proprement dit. Le décorateur - ou plutôt, le directeur artistique Félix Murcia ${ }^{1}$ - tente avec lucidité cette définition de son métier:

La dirección de arte es una de las especialidades no específicas del cine. Su principal contenido es lograr la verosimilitud de los escenarios, y se sirve, para ello, de la escenotecnia como recurso tanto técnico como artístico. También debe dotar a otros escenarios, ya sean naturales (paisajes), artificiales

1 Félix Murcia, né en 1945 à Aranda de Duero (Burgos), est diplômé des Arts et Métiers de Madrid en architecture d'intérieurs, arts appliqués, scénographie et design. Il a travaillé comme décorateur-adjoint auprès de Rafael Palmero, notamment pour les deux derniers épisodes de La Forja de un rebelde. C'est le directeur artistique qui a le plus remporté de Goyas (cinq) pour les décors de cinq films d'époque. 
preexistentes (edificaciones), construidos artificialmente (decorados), o adaptados (paisajes y edificaciones reales y decorados artificiales), tanto de funcionalidad como de expresividad, desde las exigencias que plantea cualquier acción dramática que se pretenda filmar. (Murcia 2002: 45)

Juan Pedro de Gaspar, directeur artistique de El Lápiz del carpintero et de Deseo, nous donne cette réponse lorsque nous lui demandons de préciser les limites de son métier:

Por mi experiencia, en España está algo desdibujada la frontera entre los distintos oficios del cine. Las atribuciones están más definidas en el cine norteamericano. Aquí, en ausencia de production designer, el director de arte asume las funciones de concepción visual de la película y diseño de decorados, tanto construidos como localizaciones, haciendo dibujos de concepto y desarrollando luego la construcción con los ayudantes y trabajando con el decorador, que asumiría las funciones de set designer, set decorador y set dresser, en el «acabado $»^{2}$.

La contribution du directeur artistique, selon Juan Pedro de Gaspar, va donc clairement au-delà de celle du simple travail de construction de décors. L'idée de "conception visuelle du film» suppose que la création des décors se fait en symbiose avec le reste de l'équipe pour obtenir le résultat le plus satisfaisant possible. Elle donne au directeur artistique un nouveau statut, passant de celui d'artisan (architecte, charpentier, etc.) à celui d'artiste et de créateur et cela induit une reconnaissance accrue de son travail. En somme, comme le souligne Jean-Pierre Berthomé,

L'art du décorateur n'est pas facile à cerner. Et cela, d'abord, parce que sa contribution au film doit idéalement fusionner avec d'autres tout aussi essentielles qui font que le résultat final, dans la plupart des cas, tient à une addition d'apports dont aucun n'est séparable des autres. Cette interdépendance est spécialement évidente entre le décorateur qui crée les espaces de la fiction et le chef opérateur qui s'en empare pour les transformer en images qui n'auront de réelle existence que dans la lumière de la projection sur l'écran (Berthomé 2003: 17).

Félix Murcia met d'abord l'accent sur la question de la vraisemblance des décors qui doivent obtenir l'adhésion des spectateurs. On touche là à un sujet essentiel de la reconstitution historique puisque la vérité, si tant est qu'il soit possible de la connaître, reste insaisissable. Afin de ne pas trop nous écarter de notre propos, rappelons simplement que, pour Paul Ricœur, la vérité est intangible et il ne peut y avoir de compromis avec elle. Mais le philosophe concède que le récit historique, pour sa part, aura immanquablement

2 Entretien de l'auteur avec Juan Pedro de Gaspar, du 16 décembre 2005. 
une part de fiction pour combler les «blancs» (Ricœur 1985: 338). Sur la question de l'histoire et de la vérité, Tzvetan Todorov va plus loin dans la perception que nous aurons du passé selon le récit qui en est fait. Pour lui, il existe deux régimes de vérité, une vérité des faits, indiscutables et établis par le croisement des sources, qui ne peut faire l'objet de débat sauf à verser dans le révisionnisme. Et il existe aussi une vérité de dévoilement de ces faits qui est subjective car l'historien, ou l'artiste qui s'empare de l'histoire pour la fictionnaliser, vont se placer dans une situation délicate dans la mesure où leur subjectivité peut nourrir la critique d'une falsification sous quelque forme que ce soit (Todorov 1989: 10). C'est un débat entre historiens qui concerne cependant le cinéma historique car la perception visuelle du passé va passer, pour le plus grand nombre, par la fiction cinématographique.

Le directeur artistique va donc devoir, dans le cadre du scénario, « réinventer la réalité » selon le mot de l'architecte Jorge Gorostiza (Gorostiza 2005: 25), en s'efforçant de travailler au plus proche de ce qu'il pense être la réalité. Cela passe par un travail de documentation qui est plus ou moins important selon les manières de travailler des décorateurs, mais les sources visuelles sont limitées et seront souvent récurrentes. Cela va des photographies aux documentaires mais les films de fictions des années du franquisme seront aussi utilisés pour les films historiques actuels. La célèbre photo, attribuée à David Seymour, plus connu sous son psseudonyme Chim, de la banderole madrilène "No pasarán », qui apparaît aussi dans des films d'archives, sert à créer le décor de Las bicicletas son para el verano (Jaime Chávarri, 1984). Les auteurs de films historiques aiment souvent faire écho à des tableaux de maîtres, comme José Luis Garci dans Historia de un beso (2002), même si l'époque représentée ici est antérieure à la guerre civile; dans ce film, le spectateur pourra longuement contempler des allusions transparentes à certaines toiles peintes sur la plage par Joaquín Sorolla en 1909, de véritables mises en mouvement de l'image fixe; parmi elles, on reconnaît ainsi aisément le Paseo a orillas del mar et le Niño jugando con un barco. Les emprunts seront parfois plus originaux comme dans le cas de El Florido Pensil (2002) où la bande dessinée Roberto Alcázar y Pedrín est transposée de manière décalée à l'écran, cas intéressant d'intermédialité.

Mais l'esprit de réinvention réaliste du passé sera dominant dans les fictions historiques qui vont décliner les quatre types de décors définis par Félix Murcia - paysages, édifices existants, décors construits ou un mélange des trois - en privilégiant ce que l'on pourrait identifier comme les lieux du franquisme, lieux de mémoire de cette époque, qui ne se limitent pas au domaine spatial. Des lieux qui apporteraient ainsi par leur seule apparition 
à l'écran le cachet d'authenticité recherché. Un exemple emblématique pourrait être le Valle de los Caídos, qui apparaît à trois reprises ${ }^{3}$ dans Espérame en el cielo, d'Antonio Mercero (1987), Los Años bárbaros, de Fernando Colomo (1998) et El Florido Pensil, de Juan José Porto (2002).

Le travail des décorateurs, de plus en plus apprécié à sa juste valeur, est confié à des artistes et non plus seulement à des artisans. Cela explique que certains noms reviennent bien souvent, voire certaines équipes, décorateurs, accessoiristes, costumiers, chefs-opérateurs, comme s'il s'agissait de spécialistes de la représentation du passé qui constituerait une branche spécifique de la direction artistique. Entre autres, Josep Rosell ${ }^{4}$, Gil Parrondo ${ }^{5}$, Rafael Palmero ${ }^{6}$ et Félix Murcia, ont dominé la construction de décors historiques pendant ces trente dernières années en Espagne puisqu'ils ont été directeurs artistiques de plus du quart des films historiques, avec une recréation du passé qui devient prévisible, à force d'être déclinée, à tel point que certains décors et accessoires sont partiellement réutilisés d'un film à l'autre comme nous le verrons plus loin. Ces ressemblances, même si chaque décorateur a son style propre, induisent implicitement une image très codifiée de ce passé, image d'autant plus pénétrante qu'elle est répétée dans nombre de fictions historiques. Cette cohérence et ces conventions tacites ont pour effet que les décors deviennent des modes prégnants de transmission d'une idée de l'histoire et peuvent être considérés comme des codes eux-mêmes. Cela est confirmé par Gil Parrondo: «cada época tiene tal fuerza que le obliga a uno seguir un poquito la pauta de cada momento » (Fernández Mañas 1997: 24). Les décorateurs que nous avons cités ont un

3 Kevin Perromat nous signale aimablement que le Valle de los Caídos apparaît également dans Balada triste de trompeta d'Álex de la Iglesia (2010) mais il s'agit dans ce cas d'un usage totalement décalé de la référence réaliste.

4 Josep Rosell Pal, né en 1950 à Tremp (Lérida) a étudié le dessin industriel à Barcelone puis a travaillé comme dessinateur de meubles de style avant de rejoindre l'atelier du peintre Jaume Xifra à Paris. On lui a confié la direction artistique d'une douzaine de films espagnols.

5 Gil Parrondo, né en 1921 dans les Asturies, a commencé à travailler en 1939 comme assistant-décorateur de Sigfrido Burmann puis, dès 1952, comme chef-décorateur. Il a élaboré les décors d'une centaine de films, parfois sans apparaittre au générique (ce fut le cas pour Mr. Arkadin d'Orson Welles). Il est le seul Espagnol à avoir obtenu deux Oscars du meilleur décor pour deux films historiques de Franklin J. Shaffner, Patton en 1970, puis Nicholas and Alexandra, en 1971. Il a été D.A. pour une dizaine de films historiques en Espagne sans compter les nombreuses productions hollywoodiennes auxquelles il a été associé à d'autres production designers.

6 Rafael Palmero, né en 1944 à Madrid et décédé en 2006, a commencé à travailler en 1976 pour Cría Cuervos, de Carlos Saura. Il a notamment travaillé sur ¡Ay Carmela! 
style et une façon de travailler qui leur est propre mais leurs ressemblances sont plus frappantes que leurs différences. Si Josep Rosell est un décorateur qui a tendance à l'élaboration de décors peu encombrés, confinant parfois à l'épure, où la patine n'est pas oubliée, Gil Parrondo construit des décors plus complexes, très fournis en accessoires et d'une propreté impeccable. Le résultat de leur travail est conditionné par l'éclairage et la photographie du chef-opérateur, par ailleurs souvent le même (José Luis Alcaine et Juan Amorós avec Rosell et Raúl Pérez Cubero avec Parrondo) mais ils sont toujours soumis au scénario sur lequel ils n'ont qu'une faible marge de manœuvre. On comprend ainsi que le ton des films roselliens, très amers des années 1980-1990 (La Plaza del Diamante, Tiempo de silencio, Amantes...), soit très différent, voire divergent, de la plupart des films parrondiens, assez nostalgiques voire doucereux pour ceux tournés et produits par José Luis Garci autour de l'an 2000 (You're the one, Historia de un beso, Tiovivo c.1950...).

Pourtant, les ingrédients de la fiction historique se retrouvent bel et bien chez ces deux importants décorateurs, comme chez les autres, et il est intéressant de constater combien le mimétisme les touche au point de réutiliser des décors existants dans des films sans rapport entre eux. Ainsi, pour figurer les ruines du village de Belchite dans ;Ay, Carmela!, le décorateur Rafael Palmero réemprunte en 1990 des décors qu'il avait créés en 1988 pour la série télévisée La forja de un rebelde, dont l'action se déroulait à Madrid fin 1936, tournée par Mario Camus d'après l'œuvre de l'écrivain exilé Arturo Barea.

Le caractère interchangeable des ruines, donne une idée universelle de celles-ci et elles renvoient aux photos de Capa, de Gerda Taro, de Chim ou d'autres photo-reporters de la guerre civile. Cela induit aussi une réflexion sur la trace de ce qu'il en reste et la perception que nous en avons puisque l'étendue des dommages reste à imaginer ou bien semble limitée à ce que nous en voyons à l'écran. Paul Ricœur nous dit que c'est dans le "phénomène de la trace que culmine le caractère imaginaire des connecteurs qui marquent l'instauration du temps historique». À ces traces, dit-il, «on n'assigne leur valeur d'effet signe qu'en se figurant le contexte de vie (...) bref, le monde qui aujourd'hui manque, si l'on peut dire autour de la relique» (Ricœur 1985: 335).

Josep Rosell va, pour sa part, réutiliser quasi tel quel le décor d'un espace récurrent, la façade d'un cinéma, dans trois films différents, La Plaza del Diamante, Si te dicen que caí et El Crimen del Cine Oriente. Ce réemprunt, 
opéré par Rosell à lui-même, s'il peut se justifier par des questions de coût, a l'inconvénient d'induire là encore une perception quelque peu biaisée dans la mesure où l'on pourra dégager l'impression que tous les cinémas étaient semblables à celui-là sous le franquisme. Au moins, Rosell a-t-il maquillé le cinéma qui n'est pas si facilement reconnaissable. Ce n'est pas le cas du prestigieux décorateur Gil Parrondo qui a réutilisé un décor à peine retouché pour deux films de José Luis Garci, You're the one (2000) et Historia de un beso (2002), décor qu'il avait créé pour El abuelo (1998). La route qui mène le spectateur vers l'entrée du village fictif de Cerralbos del Sella est en tout point semblable d'un film à l'autre, de même que la cour d'école, le café ou encore la maison de maître entièrement modelée par Gil Parrondo. L'affiche «Nitrato de Chile», visible à l'entrée du village, est aussi un accessoire récurrent des films historiques. Dans la cuisine de la maison, tout est strictement à la même place, dans le salon, le mobilier est rigoureusement identique. Parrondo, sur les indications du metteur en scène, n'a que très légèrement modifié certains détails car il y a, chez José Luis Garci, une volonté délibérée de figer le passé dans une imagerie volontiers nostalgique et esthétisante, qui correspond à des motivations que l'on peut soupçonner d'être idéologiques, aspect qu'il serait intéressant de développer par ailleurs.

Observons encore deux exemples, cette fois concernant les accessoires, qui sont également la responsabilité des D.A., nous voulons parler des véhicules de transport. Objet incontournable, c'est surtout la voiture que l'on retrouve d'un film à l'autre, toujours rutilante, et par là même quelque peu fétichisée. Mais on trouve aussi d'autres types de véhicules et, dans ce cas, le réemprunt devient quasi inévitable. Le tramway et la publicité pour le savon «El Oso» que nous apercevons dans La Mujer del anarquista (M. Noëlle, P. Sehr, 2008) ne nous rappelle-t-il pas celui de Las Trece Rosas (E. Martínez Lázaro, 2007)? Ou encore Tranvía a la Malvarrosa (L. García Sánchez, 1996)? Dans le même ordre d'idées, l'autocar placé par le décorateur Josep Rosell dans trois films différents (El viaje a ninguna parte, El Año de las luces, Amantes) remplit une fonction de preuve de l'ancrage historique. En voyant le car, le spectateur rattache le film à un ensemble de signes d'historicité indiscutable. La récurrence de la présence de l'objet s'inscrit dans un vaste et complexe système des objets dans les films historiques, comme aurait pu le théoriser Abraham Moles - pour qui l'objet était aussi vecteur de communication (Moles 1972: 8) - système qui semble vouloir que figurent obligatoirement, un ensemble donné d'accessoires, ensemble qui va bien entendu varier légèrement d'un film à l'autre, mais sans lequel le 
film ne serait pas reçu, pas accepté comme vraisemblable, trop éloigné des représentations mentales actuelles de ce qu'était le passé. La non-observance de cette inclusion d'accessoires pourrait également empêcher de satisfaire la pulsion scopique nostalgique, très forte dans le cinéma américain comme l'a démontré Christine Sprengler dans Screening nostalgia, qui fait ressembler certains films historiques à des catalogues animés de musée autour desquels se greffe un récit de fiction (Sprengler 2009: 93). Le cinéma européen, et singulièrement espagnol, n'est pas à l'abri de ce penchant. Et lorsqu'un film s'affranchit de la règle non écrite de la prégnance du décor en pratiquant une épure presque absolue, comme le fit en 2009 Francisco Avizanda dans Hoy no se fía, mañana sí, l'œuvre encourt le risque d'une incompréhension quasi générale.

Pour conclure, nous pourrions nous interroger sur l'avenir qu'a le décor de film historique si l'on admet que l'on continuera à en construire malgré l'arrivée des techniques numériques. La réutilisation des décors n'est pas un phénomène nouveau et a été pratiquée partout dès les débuts du cinéma (Puaux 2008: 35). Mais, dans le cas du film historique, il nous semble que l'esthétisation, parfois le fétichisme, souvent la culture complaisante de la nostalgie et la réduction à un système de codes fonctionnant en quelque sorte en circuit fermé, avec des réemprunts nombreux, peut conduire à une lecture univoque de l'histoire. En revanche, c'est bien cette rigueur des directeurs artistiques, qui n'ont jamais été aussi bien documentés et conseillés, qui crée les conditions d'une transmission la moins altérée possible de la mémoire historique. En ce sens, les emprunts des décorateurs à d'autres arts et au cinéma, et les réemprunts à leurs propres créations, sont parfaitement légitimes.

\section{Bibliographie}

Berthomé, J.-P., 2003, Le Décor au cinéma, Paris, Cahiers du cinéma.

Blayo, N., 2010, «La chanson, un langage à part dans le cinéma espagnol», in Quand le cinéma prend la parole, Les Cahiers du Littoral, Shaker Verlag.

Fernández Mañas, I., 1997, Gil Parrondo. Pasión y rigor, Almería, Diputación de Almería.

Gaudreault, A., 1999, «Distance et historicité: problèmes de méthode de la 'reconstitution historique'», in Le Cinéma en histoire, Québec, Paris, Éditions Nota Bene/ Klincksieck. 
Gorostiza, J., 1997, Directores artísticos del cine español, Madrid, Cátedra/Filmoteca Española.

Gorostiza, J., 2001, La arquitectura de los sueños. Entrevistas con directores artísticos del cine español, Madrid, Festival de Cine de Alcalá de Henares/Fundación Colegio del Rey/Consejería de las Artes de la Comunidad de Madrid/Institut Valencià de Cinematografia Ricardo Muñoz Suay.

Lukacs, G., 1965, Le roman historique, Paris, Payot.

Moles, A., 1972, Théorie des objets, Paris, Éditions universitaires.

Murcia, F., 2002, La escenografía en el cine. El arte de la apariencia, Madrid, Fundación Autor, SGAE.

Puaux, F., 2008, Le décor de cinéma, Paris, Cahiers du cinéma.

Ricœur, P., 1985, Temps et récit, III. Le temps raconté, Paris, Seuil.

Sprengler, C., 2009, Screening nostalgia. Populuxe props and Technicolor aesthetics in contemporary American films, New York/ Oxford, Bergham Books.

Todorov, T., 1989, «Fictions et vérités» L’Homme, tome 29 n 111-112. Littérature et anthropologie, p. 7-33.

Filmographie

Aranda, V., 1991, Amantes.

Aranda, V., 1989, Si te dicen que caí.

Aranda, V., 1986, Tiempo de silencio.

Avizanda, F., 2009, Hoy no se fía, mañana sí.

Betriu, F., 1981, La Plaza del Diamante.

Camus, M., 1990, La Forja de un rebelde.

Chávarri, J., 1984, Las Bicicletas son para el verano.

Colomo, F., 1998, Los Años bárbaros.

Costa, P, 1997, El Crimen del Cine Oriente.

Fernán-Gómez, F., 1986, El viaje a ninguna parte.

Garci, J. L., 1998, El Abuelo.

Garci, J. L., 2002, Historia de un beso.

Garci, J. L., 2004, Tiovivo c.1950.

Garci, J. L., 2000, You're the one.

García Sánchez, L., 1996, Tranvía a la Malvarrosa.

Martínez Lázaro, E., 2007, Las Trece Rosas.

Mercero, A., 1987, Espérame en el cielo.

Noëlle, M., \& Sehr, P., 2008, La Mujer del anarquista.

Porto, J. J., 2002, El Florido Pensil.

Reixa, A., 2003, El Lápiz del carpintero.

Saura, C., 1990, ¡Ay, Carmela!

Trueba, F., 1986, El Año de las luces.

Vera, G., 2002, Deseo. 